\title{
Current concepts in the diagnosis and management of autoimmune hepatitis
}

\author{
Stephen Kriese, Michael A Heneghan
}

Institute of Liver Studies, King's College London Medical School at King's College Hospital, London, UK

\section{Correspondence to}

Dr Michael Heneghan, Institute of Liver Studies, King's College London Medical School at King's College Hospital, Bessemer Road, London SE5 9RS, UK; michael.heneghan@nhs.net

Received 17 May 2012 Revised 6 July 2012 Accepted 8 July 2012
To cite: Kriese $S$, Heneghan MA. Frontline Gastroenterology 2013, 4, 2-11.

\begin{abstract}
Autoimmune hepatitis (AlH) is a progressive necroinflammatory liver disease associated with significant morbidity and mortality. Mainly affecting females, AlH has a varied clinical presentation from minor symptomatology to acute liver failure. The diagnosis should be considered in anyone with abnormal liver function tests. Diagnostic features include biochemical evidence of transaminitis, elevated $\lg$ G and positive autoantibodies. Liver biopsy may show interface hepatitis with portal-based plasma cell infiltrates. A clinical and pathological spectrum of disease exists with other autoimmune liver disease in rare cases. AlH responds promptly to immunosuppression therapy, including corticosteroids (prednis(ol)one or budesonide) with azathioprine. Treatment failure can be addressed with several second-line immunosuppressive agents. Liver transplantation remains a successful salvage therapy for acute autoimmune liver failure or treatment failure in chronic AlH complicated by synthetic dysfunction, portal hypertension or hepatocellular carcinoma.
\end{abstract}

\section{INTRODUCTION}

Autoimmune hepatitis (AIH) is a progressive necroinflammatory condition of the liver. It is rare and its incidence varies geographically. The annual incidence of AIH in Europe is $0.8-1.9$ cases per 100000 with a point prevalence of 11.6-17 per 100000 population. ${ }^{1} \mathrm{AIH}$ can be largely divided into two types based on antibody profiles which, however, overlap with other existing autoimmune liver diseases. $\mathrm{AIH}$ is a rare but important differential diagnosis of abnormal liver function tests, as untreated patients have high mortality. ${ }^{2}$

\section{PATHOGENESIS}

Fundamentally, AIH is caused by a loss of immunological self-tolerance. The reasons for this, however, remain incompletely understood. A genetic component to susceptibility is suggested by the preponderance of patients who are female, in addition to the findings of specific allotypes within the major histocompatability complex alleles. These genetic associations vary between racial and geographical populations; in Northern America and Northern Europe, the human leucocyte antigen allotypes DRB1*0301 and DRB1*0401 are associated with AIH. ${ }^{1}$

Molecular mimicry by viral agents has been suggested as a possible mechanism of loss of self-tolerance. Cross-reactive antibodies to hepatic antigens can be found in patients with $\mathrm{AIH}$, cytomegalovirus, herpes simplex virus and hepatitis C. ${ }^{3}$ Similarly, multiple drugs have been implicated in the pathogenesis in the genetically susceptible patient including minocycline, nitrofurantoin and atorvastatin. $^{5}$

Recent evidence also suggests that the adaptive and humoral immune response is dysregulated in AIH. A key area of investigation concerns regulatory $\mathrm{T}$ cells (CD4 CD25), which promote selftolerance by suppressing proliferation of auto-reactive $\mathrm{T}$ cells. These have been shown to be deficient both in number and function in AIH patients. ${ }^{6-9}$ Figure 1 summarises some of the putative factors in immunopathogenesis.

\section{DIAGNOSIS}

\section{Clinical presentation}

AIH demonstrates a female preponderance of up to $4: 1$. Classically perceived as a disease of women in early life, recent evidence suggests that $\mathrm{AIH}$ presents in all stages of life and may even be more common in individuals over the age of 60 years. ${ }^{10}{ }^{11} \mathrm{AIH}$ demonstrates a varied 


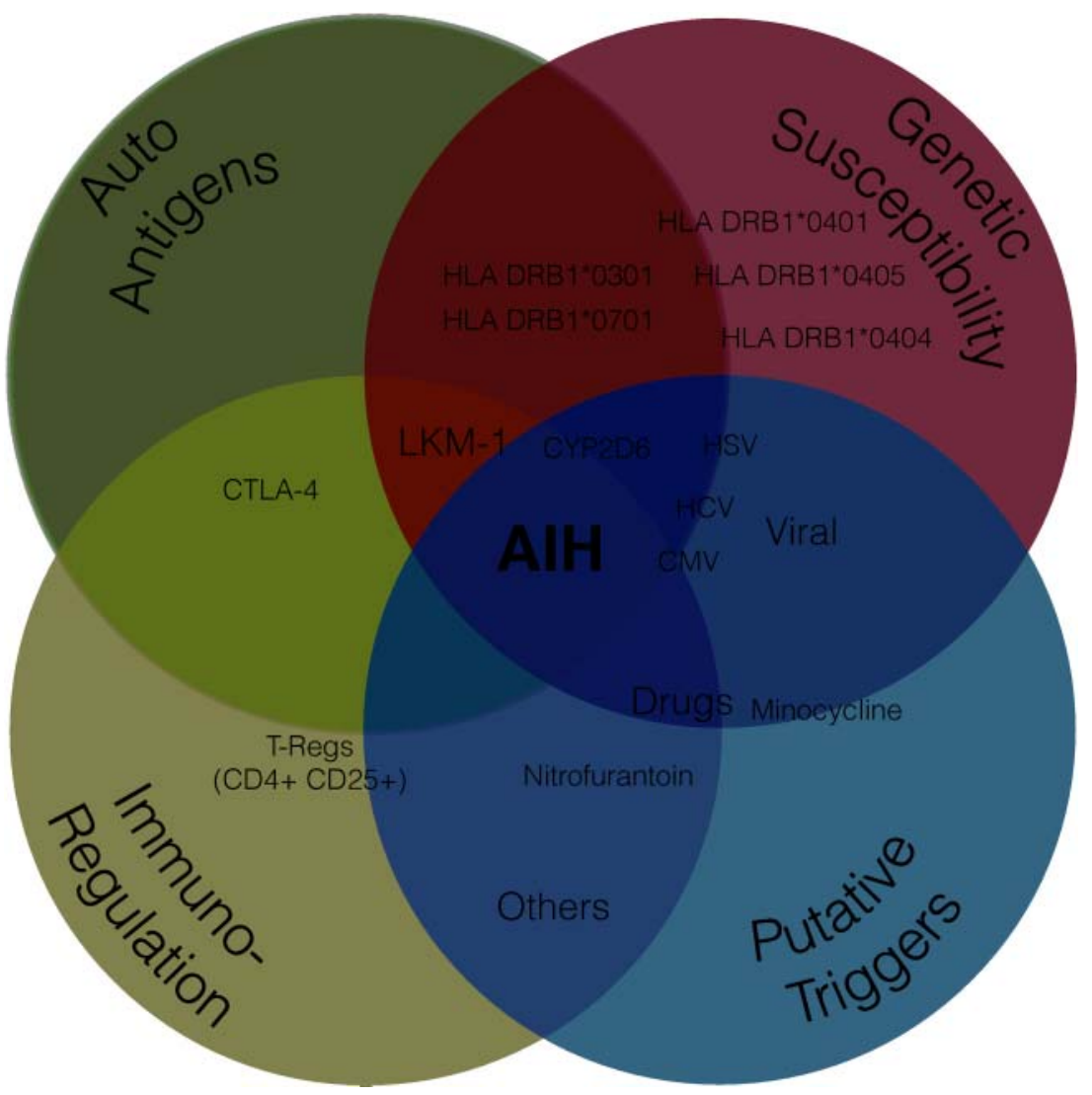

Figure 1 Immunopathogenesis of autoimmune hepatitis. AlH, autoimmune hepatitis; CMV, cytomegalovirus; CTLA-4, cytotoxic T lymphocyte-associated antigen 4; CYP2D6, member of cytochrome P450 family; HCV, hepatitis C virus; HLA, human leucocyte antigen; HSV, herpes simplex virus; LKM-1, antiliver kidney microsome 1 antibodies; T-Regs, regulatory T cells.

clinical phenotype, and is somewhat chameleonic in its presentation (figure 2). Data from a Swedish population study suggests a small number $(12 \%)$ of patients are asymptomatic at the time of diagnosis, whereas, fatigue is the most commonly reported symptom $(61 \%) .{ }^{12}$ Other associated symptoms may include arthralgia of small joints, nausea, vomiting, abdominal pain, pruritus or skin rash.

An acute icteric illness may be seen in approximately $25 \%$ of patients. ${ }^{13}$ A small subset of these patients may go on to develop acute liver failure (ALF); $2 \%-5 \%$ of transplants performed for ALF are attributable to $\mathrm{AIH} .{ }^{14-16}$

Lastly, up to a third of patients are cirrhotic at diagnosis and present with symptoms and signs of chronic liver disease, such as jaundice, ascites or variceal bleeding. ${ }^{17}$ According to some studies, advanced disease is more common in specific populations including black and elderly patients. ${ }^{10} 18$

Other autoimmune diseases are prevalent in $\mathrm{AIH}$, such as thyroiditis $(10 \%-23 \%)$, ulcerative colitis (2\%-8\%), coeliac disease (1\%-2\%), type-1 diabetes (7\%-9\%), rheumatoid arthritis (2\%-5\%) and systemic lupus erythematous $(1 \%-2 \%) .{ }^{19}$

\section{Autoantibodies}

In conjunction with persistently elevated aminotransferases and hyper-gammaglobulinaemia (in particular $\operatorname{IgG}$ ) the presence of autoantibodies is the hallmark of AIH. An expanding pool of autoantibodies has been described in AIH including antinuclear antibody (ANA), antismooth muscle (SMA), antiliver kidney

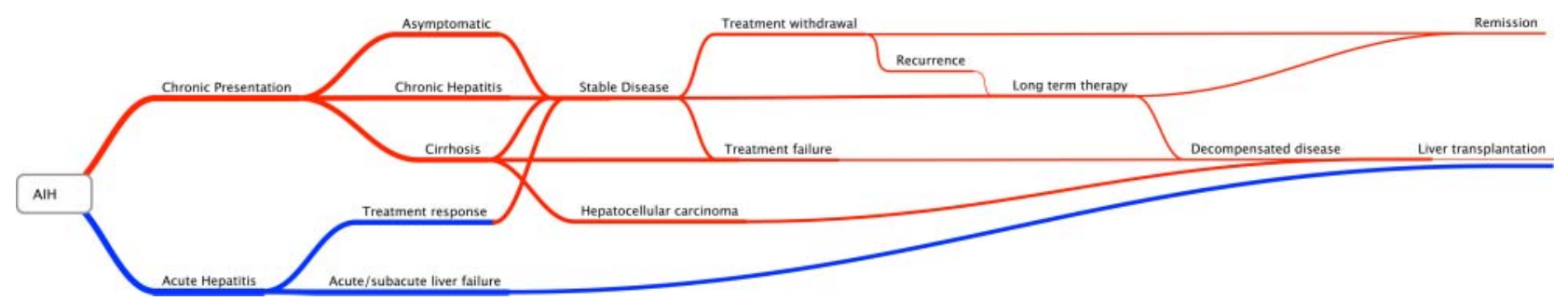

Figure 2 Modes of presentation and clinical outcome in autoimmune hepatitis. 
microsome 1 (LKM-1), antineutrophil cytoplasmic, antisoluble liver antigen/liver-pancreas (SLA/LP), antiliver cytosol 1 (LC1), anti-double-stranded DNA, antiactin, antichromatin, antiasialoglycoprotein receptor (ASGPR) and anticyclic citrullinated peptide. The roles these autoantibodies play in the pathogenesis of $\mathrm{AIH}$ are still poorly understood, although the clearest association exists with LKM-1-associated AIH. Two main types of AIH have been described by their characteristic autoantibodies profiles. Detectable ANA and SMA typify type $1 \mathrm{AIH}$, while LKM or LC1 typify type 2 . Type $2 \mathrm{AIH}$ is far rarer, more common in children, and associated with a more aggressive clinical course. $^{17}$

A recent study analysed the diagnostic utility of the conventional autoantibodies in AIH. ANA, SMA and LKM1 had sensitivities of only $32 \%, 16 \%$ and $1 \%$, respectively. The sensitivity of ANA and SMA combined increased to $43 \%$ with a positive predictive value of $97 \%$. Importantly, both ANA and SMA were seen in other liver conditions, including alcoholrelated liver disease (ALD), hepatitis $B$ and $C$ virus infection, and non-alcoholic fatty liver disease. ${ }^{20}$

Some autoantibodies have been shown to be important markers of more severe histological changes (SLA, LKM1, LC1, ASPGR , antiactin), associated with need for transplantation or liver failure (SLA, antiactin) and treatment dependence (SLA, ASGPR, antichromatin). ${ }^{21}$

\section{Histology}

Liver biopsy remains essential for the diagnosis of AIH. Although no pathognomonic histological findings exist for $\mathrm{AIH}$, the classical findings are those of an interface hepatitis with a lymphoplasmacytic infiltrate at the junction of the portal tract and hepatic parenchyma (figure 3). ${ }^{22}$ This is found in $84 \%-98 \%$ of patients, but may also occur in liver injury induced by drugs or viruses. ${ }^{23}$ Lobular necrosis can be seen in more severe acute forms of $\mathrm{AIH}{ }^{24}$

\section{Diagnostic criteria}

The International Autoimmune Hepatitis Group (IAIHG) has devised several scoring systems (tables 1 and 2) to aid in research and diagnosis in clinical practice. $^{22} 2526$ The diagnostic utility of the guidelines has been extensively studied in different ethnic and geographical populations. ${ }^{27-33}$ The 1999 scoring system is noted to be highly sensitive and specific for a definitive diagnosis of $\mathrm{AIH}$, but is cumbersome. ${ }^{31}$ The revised simplified 2008 criteria retain specificity but lack sensitivity $(99.5 \%$ and $70 \%$, respectively) (table 3). ${ }^{31}$ Those patients presenting with acute or sub-ALF can often be mischaracterised by these scoring systems, as the classical features of $\mathrm{AIH}$ are not always present. ${ }^{31} 34$

$\mathrm{AIH}$ is, in essence, a diagnosis of exclusion, and attention must be given to exclude viral-related infection, such as Hepatitis B and C. As with all diagnostic criteria, false positive and false negative results can be attained, and this stresses the importance of critically analysing each of the major components of the scoring system in conjunction with the clinical history. ${ }^{35}$

\section{The dilemma of 'overlap' syndromes and IgG4 disease}

Some patients with AIH may also present with features from the spectrum of other autoimmune liver disease, such as primary biliary cirrhosis (PBC) and primary sclerosing cholangitis (PSC). There is no standardised definition of what constitutes an overlap syndrome. The IAIHG has recently published a report clarifying their expert opinion in this area. They state that overlapping features of AIH and PSC or PBC are present between $2 \%$ and $19 \%$ of cases, but that clinicians should focus on the predominant disease phenotype and treat accordingly, rather than focusing on the idiosyncrasies of both conditions. ${ }^{36}$

\section{PBC/AlH overlap}

The European Association for the Study of the Liver have adopted criteria for diagnosis of $\mathrm{AIH} / \mathrm{PBC}$
A

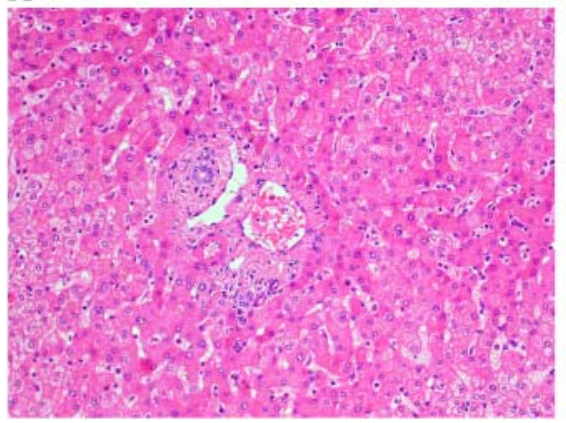

B

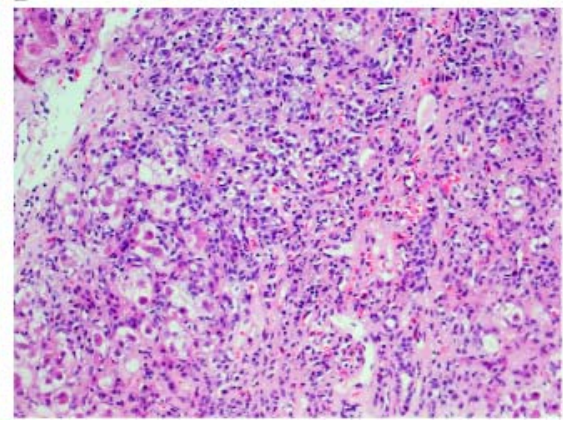

Figure 3 Classical histological features of autoimmune hepatitis $(\mathrm{AlH})$. (A) Photomicrograph of a core needle biopsy demonstrating normal liver histology. (B) Photomicrograph of core needle biopsy from a patient with AlH demonstrating an enlarged portal tract, disruption of the limiting plate with interface activity and a significant plasma cell infiltrate. Photomicrographs taken at $\times 200$ magnification and stained with $\mathrm{H} \& \mathrm{E}$. 
Table 1 Revised IAIHG diagnostic criteria (1999)

\begin{tabular}{|c|c|}
\hline Parameters/features & Score \\
\hline Females & +2 \\
\hline \multicolumn{2}{|l|}{ ALP:AST (or ALT) ratio } \\
\hline$<1.5$ & +2 \\
\hline $1.5-3.0$ & 0 \\
\hline$>3.0$ & -2 \\
\hline \multicolumn{2}{|l|}{ Serum globulins or lgG above normal } \\
\hline$>2.0$ & +3 \\
\hline $1.5-2.0$ & +2 \\
\hline $1.0-1.5$ & +1 \\
\hline$<1.0$ & 0 \\
\hline \multicolumn{2}{|l|}{ ANA, SMA or LKM1 titres } \\
\hline$>1 / 80$ & +3 \\
\hline $1: 80$ & +2 \\
\hline $1: 40$ & +1 \\
\hline$<1: 40$ & 0 \\
\hline AMA positive & -4 \\
\hline \multicolumn{2}{|l|}{ Hepatitis markers } \\
\hline Positive & -3 \\
\hline Negative & +3 \\
\hline \multicolumn{2}{|l|}{ Drug history } \\
\hline Positive & -4 \\
\hline Negative & +1 \\
\hline \multicolumn{2}{|l|}{ Average alcohol consumption } \\
\hline$<25$ g per day & +2 \\
\hline$>60$ g per day & -2 \\
\hline \multicolumn{2}{|l|}{ Liver histology } \\
\hline Interface hepatitis & +3 \\
\hline Predominantly lymphocytic infiltrate & +1 \\
\hline Rosetting of liver cells & +1 \\
\hline None of the above & -5 \\
\hline Biliary changes & -3 \\
\hline Other changes & -3 \\
\hline Other autoimmune diseases & +2 \\
\hline \multicolumn{2}{|l|}{ Optional additional parameters } \\
\hline Seropositivity for other defined antibodies & +2 \\
\hline HLA DR3 or DR4 & +1 \\
\hline \multicolumn{2}{|l|}{ Response to therapy } \\
\hline Complete & +2 \\
\hline Relapse & +3 \\
\hline \multicolumn{2}{|l|}{ Interpretation of aggregate scores } \\
\hline \multicolumn{2}{|l|}{ Pretreatment } \\
\hline Definite AlH & $>15$ \\
\hline Probable AlH & $10-15$ \\
\hline \multicolumn{2}{|l|}{ Post-treatment } \\
\hline Definite AlH & $>17$ \\
\hline Probable AlH & $12-17$ \\
\hline
\end{tabular}

Adapted from Alvarez et al. ${ }^{22}$

AlH, autoimmune hepatitis; ANA, antinuclear antibody; HLA, human leucocyte antigen; IAIHG, International Autoimmune Hepatitis Group; LKM1, anti liver kidney microsome 1; SMA, antismooth muscle.

overlap which require at least two diagnostic criteria to be present from each disease to make the diagnosis. The AIH criteria include (1) alanine aminotransferase
Table 2 Simplified diagnostic criteria for $\mathrm{AlH}$

\begin{tabular}{lll}
\hline Variable & Cut-off & Points \\
\hline ANA or SMA & $>1: 40$ & 1 \\
ANA or SMA or LKM & $>1: 80$ & 2 \\
SLA & Positive & 2 \\
IgG & $>$ ULN & 1 \\
& $>1.1$ ULN & 2 \\
Liver histology & Compatible with AlH & 1 \\
& Typical AlH & 2 \\
\hline
\end{tabular}

Score $>6$ probable $\mathrm{AlH}>7$ definite $\mathrm{AlH}$.

From Hennes et al. ${ }^{26}$

AlH, autoimmune hepatitis; ANA, antinuclear antibody; LKM, antiliver kidney microsome; SLA, soluble liver antigen; SMA, antismooth muscle; ULN, Upper limit of normal.

levels at least five times the upper limit of normal (ULN); (2) IgG levels at least two times that of ULN, or positive SMA and (3) a liver biopsy with moderate or severe periportal or interface lymphocytic hepatitis. The $\mathrm{PBC}$ criteria include (1) alkaline phosphatase (ALP) levels at least twice that of ULN, or $\gamma$-glutamyl transferase at least five times that of ULN; (2) positive anti-mitochondrial antibody (AMA) and (3) a liver biopsy demonstrating a florid bile duct lesion. ${ }^{37}$ Features of AIH have been described to be present at diagnosis of $\mathrm{PBC}$ and also to develop over time. Whether or not PBC-AIH overlap is associated with a different prognosis to the primary disorders, if controversial, and no controlled studies exist for the treatment of PBC-AIH syndromes. ${ }^{36}$

\section{AIH/PSC overlap}

When the IAIHG criteria are applied to PSC patients $7 \%-14 \%$ of cases may have features of $\mathrm{AIH}{ }^{36}$ Alternatively, patients with AIH may be discovered to have a cholangiopathy at imaging, however, the IAIHG would argue that these cannot be truly classed as an overlap, as cholangiography has often not been performed at initial diagnosis for comparison. PSC-AIH overlap seems to be more common in

Table 3 Sensitivity, specificity, PPV, and NPV of the Simplified and 1999 IAIHG Criteria for diagnosis of AlH

\begin{tabular}{lccrc}
\hline Criteria & $\begin{array}{l}\text { Sensitivity } \\
(\%)\end{array}$ & $\begin{array}{l}\text { Specificity } \\
(\%)\end{array}$ & $\begin{array}{r}\text { PPV } \\
(\%)\end{array}$ & $\begin{array}{l}\text { NPV } \\
(\%)\end{array}$ \\
\hline Simplified criteria & & & & \\
$\quad$ Probable AlH (6-7) & 90 & 98 & 97 & 92 \\
$\quad$ Definite AlH (>7) & 70 & 100 & 100 & 74 \\
$\quad$ Overall disagnosis AlH (>6) & 90 & 98 & 97 & 92 \\
Revised 1999 criteria & & & & \\
$\quad$ Probable AlH(10-15) & 100 & 97 & 96 & 100 \\
$\quad$ Definite AlH (>15) & 99 & 98 & 97 & 99 \\
Overall disagnosis AlH (>10) & 100 & 97 & 97 & 99 \\
\hline Adpted from Yoman et & & & &
\end{tabular}

Adapted from Yeoman et al. ${ }^{31}$

AlH, autoimmune hepatitis; IAIHG, International Autoimmune Hepatitis

Group; NPV, negative predictive value; PPV, positive predictive value. 
children where it has been extensively studied and has an apparent better response to immunosuppressive therapy than PSC. ${ }^{38}$

\section{IgG4-related systemic disease and AlH}

Once thought to be an organ-specific autoimmune disease, study of IgG4-related autoimmune pancreatitis (AIP) led to the implication of IgG4 in extrapancreatic lesions in numerous target organs including the liver. ${ }^{39}$ Some groups have investigated whether a subgroup of AIH exists that is associated with IgG4. Abnormally high levels of IgG4 may be present in immunostaining of biopsy specimens in $2 \%-34 \%$ of cases depending on the stringency of the histological criteria used. In contrast with AIP, cholangiopathy is absent in IgG4-related $\mathrm{AIH}$, and it seems to demonstrate a prompt response to corticosteroid therapy. ${ }^{40}{ }^{41}$ However, case series are limited, and the significance of IgG4-related disease in AIH remains uncertain.

\section{MANAGEMENT}

\section{Pharmacological therapy}

Definitive indications for treatment have been developed on the basis of three randomised control trials that demonstrated features associated with poor outcome. They form the basis for the American Association of the Study of Liver Disease (AASLD) and the British Society of Gastroenterology (BSG) practice guidelines. ${ }^{19}{ }^{42}$ These include a serum aspartate transaminase (AST) of a least 10 times the ULN or five times the ULN, with a $\gamma$-globulin level more than twice the ULN and, lastly, the histological findings at diagnosis of bridging necrosis or multilobular necrosis. Current standard therapy is azathioprine alone, or in combination with prednis(ol)lone. However, recent studies have highlighted some potential important alternative treatments.

\section{Induction and maintenance of remission}

The mainstay of induction of remission remains corticosteroids with or without the addition of azathioprine. A recent systematic review of randomised control trials since the $1950 \mathrm{~s}$ confirms that prednis(ol)one monotherapy, or preferably in conjunction with azathioprine, remains an effective induction regimen. ${ }^{43}$

Variance exists in the induction dose of steroid from $30 \mathrm{mg} / \mathrm{d}$ to $60 \mathrm{mg} / \mathrm{d}$. A common starting dose is $40 \mathrm{mg} / \mathrm{d}$. Azathioprine $(1 \mathrm{mg} / \mathrm{kg}$ per $24 \mathrm{~h})$ can be added at the start of induction or after normalisation in liver biochemistry has been documented, as azathioprine can be associated with an idiosyncratic drug-related hepatitis. ${ }^{44} 45$

Maintenance therapy with low-dose prednis(ol)one $(<10 \mathrm{mg}$ per $24 \mathrm{~h})$ and azathioprine, or azathioprine monotherapy at higher dose $(2 \mathrm{mg} / \mathrm{kg}$ per $24 \mathrm{~h})$, is more effective than prednis(ol)one monotherapy. ${ }^{43} 44$
A recent multicentred randomised control trial has demonstrated that budesonide, a glucocorticosteroid with a high affinity for the glucocorticoid receptor, at a dose of $9 \mathrm{mg} / \mathrm{kg}$ per $24 \mathrm{~h}$ in conjunction with $1-2 \mathrm{mg} / \mathrm{kg}$ of azathioprine was more effective than prednis(ol)one and azathioprine at inducing remission. ${ }^{46}$ In addition, it has a high first-pass metabolism which limits its systemic side effects, but also limits its usefulness in cirrhotic patients because of portosystemic shunting. ${ }^{46}$ Long-term follow-up data is currently lacking, and there have been reports of relapse on budesonide/azathioprine maintenance. ${ }^{47}$

\section{Treatment goals, treatment withdrawal and relapse}

Whereas early trials in AIH therapy focused on reducing the aminotransferase activity to within 1-2 times ULN, more recent data suggests that our treatment goal should be the normalisation of transaminases, $\gamma$-globulins and, ideally, histological evidence of quiescent disease. Normalisation of biochemical parameters is associated with lower rates of relapse, progression to cirrhosis and more favourable outcomes. ${ }^{48-50}$

Treatment for AIH may be associated with significant side effects. Withdrawal of treatment should be considered for all patients after demonstrating a period of normal biochemical parameters and liver tissue examination, although no consensus exists in relation to how or when this can be achieved. Relapse is indicated by the increase of the AST/ALT(alanine aminotransferase) to three times ULN, or $\gamma$-globulin by two times ULN indicating recurrence of interface hepatitis. ${ }^{51}$ Rates of relapse are variable in studies but can be as high as $80 \%$ after 1 year of treatment. ${ }^{48}$ However, 2-4 years of maintenance therapy may reduce this risk to around $30 \% .{ }^{48}{ }^{52}$ Relapse may be predicted by shorter treatment duration, slow response to therapy, persistently abnormal biochemistry and the presence of LKM1 or SLA/LP antibodies. ${ }^{52-54}$

\section{Alternative treatment strategies}

Treatment failure inevitably does occur in some patients. A number of second-line agents have been explored in AIH.

\section{Mycophenelate mofetil}

Mycophenelate mofetil (MMF) is an inosine monophosphate inhibitor with both anti-T and -B cell proliferation effects. ${ }^{55}$ Historically, MMF has been used in patients with refractory $\mathrm{AIH}$, or who are intolerant of azathioprine. There have been 11 retrospective small case series published on the use of MMF for this indication with remission rates reported from $25 \%$ to $100 \%{ }^{56}$ A recent prospective single-centre case series has been published on the use of MMF for induction and maintenance treatment in naïve $\mathrm{AIH}$ patients. Fifty-nine patients were enrolled in the study and were maintained on a dose of between 1.5 and $2 \mathrm{~g}$ 
MMF per $24 \mathrm{~h}$. A complete biochemical response was reported in $88 \%$ of patients. ${ }^{57}$ Long-term safety data is lacking in MMF, and it is contraindicated in pregnancy, limiting its use in young females.

\section{Ciclosporin}

The calcineurin inhibitor, ciclosporin, has been studied only in small single-centre case series. In children, small case series have demonstrated high rates of biochemical remission, the largest of which was a multicentre prospective trial in 32 children in which 94\% achieved biochemical remission at 12 months. ${ }^{58}$ From organ transplant experience, Ciclosporin carries significant long-term side effects, including hypertension, renal insufficiency, hyperlipidaemia, hirsuitism, infection and malignancy.

\section{Tacrolimus}

Tacrolimus, also a calcineurin inhibitor, has been assessed for its utility in AIH in a small number of case series. Van Thiel et al reported an open label study of 21 patients started at $6 \mathrm{mg} / 24 \mathrm{~h}$ of Tacrolimus at induction which was associated with improvements in aminotransferase activity and also with deterioration in renal function. ${ }^{59} \mathrm{~A}$ single-centre study of seven patients demonstrated high rates of remission using Tacrolimus as induction therapy. ${ }^{60}$ The utility of Tacrolimus has also been studied for patients with refractory $\mathrm{AIH}$ in small case series with varying degrees of response reported. ${ }^{61-63}$ Tacrolimus remains an attractive option at low dose for refractory $\mathrm{AIH}$ in women planning pregnancy, as experience in liver transplant recipients has demonstrated a favourable safety profile. ${ }^{64-66}$

\section{Other immunomodulatory agents}

Further small case series have been reported in $\mathrm{AIH}$ with methotrexate, rituximab and Abatacept, but as yet have an undefined clinical role. ${ }^{67-70}$

\section{Long-term management}

\section{Long-term survival, cirrhosis and hepatocellular carcinoma}

Prognostic information from clinical series is variable. A large retrospective study of AIH patients in Canada demonstrated 10-year survival rates to liver-related endpoints of death or liver transplantation were 94\% in non-cirrhotic versus $67 \%$ in cirrhotic patients respectively. ${ }^{71}$ A recent UK study reported survival data of 245 individuals collected between 1977 and 2007 with a median follow-up period of 9.4 years. They reported increased rates of death from all-cause mortality with a standardised mortality rate 1.63 (95\% CI 1.25 to 2.02 ) as well as from complications of liver disease. Regression analysis suggests that the presence of cirrhosis, $>4$ relapses per decade and failure to normalise aminotransaminases within 12 months were risks factors for liver-related death or transplantation. $^{72}$
Approximately 30\%-50\% of $\mathrm{AIH}$ patients will develop cirrhosis. ${ }^{19}$ Risk factors for progressive disease include those with cirrhosis, failure to halve the level of AST in the first 6 months of treatment, failure to achieve persistent remission, and those of African-American descent. ${ }^{18} 537173$

Hepatocellular carcinoma (HCC) has been traditionally thought to be a rare complication of AIH. Case series from America, Japan and the UK suggest HCC is more common than previously thought. ${ }^{74-77}$ In a study from our own institution, HCC occurred at an incidence of $1.1 \%$ per year suggesting that HCC screening remains of paramount importance in cirrhotic AIH. ${ }^{74}$

\section{Liver transplantation}

Liver transplantation remains a life-saving procedure for individuals with AIH who present with ALF or decompensated cirrhosis. Analysis of the European Liver Transplant Registry data demonstrates a 5-year survival of $76 \%$ post-transplant, which is comparable with ALD but inferior to that of PBC. ${ }^{78}$ Similar survival rates have been reported in the USA. ${ }^{79}$

In patients presenting with acute icteric $\mathrm{AIH}$, a subset of patients may develop ALF and require emergency liver transplantation. Predictors of corticosteroid treatment failure in a study from our own institution highlighted higher serum bilirubin levels, higher Model of End Stage Liver Disease scores at presentation, and failure to lower UK End Stage Liver Disease score by two points at day seven of treatment as being predictive of treatment failure. ${ }^{80}$ Some authors have suggested that the increased risk of sepsis associated with intravenous steroid treatment in patients with autoimmune ALF may jeopardise their chances of successful transplant and should be avoided. ${ }^{81}$ Indications for transplantation for AIH cirrhosis are identical to those for other chronic liver diseases. ${ }^{82}$ Patients who develop ascites or variceal bleeding should be referred early to a liver transplantation centre. ${ }^{19}$

\section{Unwanted effects of therapy}

The unwanted effects of corticosteroids are well known and include weight gain, hypertension, diabetes, Cushingoid features, psychosis, osteopaenia and cataracts. Budesonide, on the basis of recent evidence, could offer a potential alternative for those patients at most risk of steroid-related unwanted effects.

Azathioprine may be associated with side effects in up to $25 \%$ of patients, and the side effects are more common in cirrhotic patients. ${ }^{19}$ While nausea is perhaps the most common, what concerns many patients and prescribers alike is the reported increased risk of malignancy and myelotoxity. The long-term malignancy risk in azathioprine use is poorly defined in $\mathrm{AIH}$. A recent nested control study in inflammatory bowel disease (IBD) patients treated with azathioprine 
for at least 1 year demonstrated an increased risk of ultraviolet light-associated non-melanoma skin cancer with an OR of $4.3(95 \%$ CI 3.1 to 6.0$){ }^{83}$ The risk of non-cutaneous cancer with azathioprine treatment outside of the setting of organ transplant is controversial. One meta-analysis study reported a fourfold increase in the risk of lymphoma in patients with IBD treated with azathioprine. ${ }^{84}$ However, in another recent meta-analysis, and along with a large retrospective cohort study of 17000 IBD patients, no increased risk was demonstrated. ${ }^{85-87}$ As IBD may also confer additional risk of lymphoma, it is difficult to extrapolate fully to $\mathrm{AIH}$, but the risk would appear to be low.

Azathioprine is a pro-drug that is, enzymatically converted to numerous active metabolites. Although the exact mechanism of the action of many of the metabolites is poorly understood, the therapeutic benefits are thought to be derived from thioguanine nucleotide metabolites (TGN) and toxic effects from methylmercaptopurine nucleotides (MeMPNs). ${ }^{88}$ Much research has focused on the prediction of toxic effects of azathioprine by assessing one of the major enzymes responsible for its metabolism, thiopurine methyltransferase (TPMT). Allelic variations of TPMT are common and result in varying degrees of enzymatic activity. Approximately $88 \%$ of the population has normal or high activity, $11 \%$ intermediate activity and $0.5 \%$ low activity. ${ }^{89}$ While TPMT phenotyping and genotyping is recommended to detect the $1: 300$ of the population homozygous for mutations that result in very low TPMT activity and high risk of myelotoxicity, in all other patients, TPMT activity testing is poorly predictive of toxicity. ${ }^{19} 9091$ More recent research in azathioprine metabolism has focused on therapeutic monitoring, with recent studies suggesting measurement of TGN, and MeMPN levels may help predict treatment response and toxicity potential irrespective of TPMT activity/genotyping. ${ }^{88} 92$

\section{AlH and pregnancy}

As AIH can often affect women of childbearing age, an important aspect of treatment is one of management of pregnancy where both patients and medical professionals have concerns regarding safety and the use of immunosuppressants. Initial reports from the 1970s suggested high rates of foetal loss and obstetric complications. ${ }^{93}$ Since then, five international case series have been published. ${ }^{94-98}$ The experience of our own institution is that of 81 pregnancies in 51 women followed-up at King's College Hospital between 1982 and 2009, median age at conception was 26 (range 1642 ), and $41 \%$ women were cirrhotic at the time of pregnancy. ${ }^{98}$ The live birth rate was $73 \%$, but was significantly lower in those women who were cirrhotic. While flares of inflammatory activity were common (33\%), serious maternal events within 12 months of pregnancy, including decompensation, need for liver transplantation or death occurred in only $11 \%$ of pregnancies. Most patients were on therapy for AIH at the time of conception, but this did not seem to impact on rates of miscarriage or foetal malformations. ${ }^{98}$ The low rate of foetal malformations in our study is also reflected in a larger study of pregnancy in IBD patients. ${ }^{99}$ AASLD practice guideline recommend discontinuation of azathioprine during pregnancy. ${ }^{42}$ However, experience of azathioprine use in our study was associated with lower rates of flares of $\mathrm{AIH}$, and those patients with suboptimal disease control, or who were on no therapy, were more likely to have high-risk pregnancies. $^{98}$ BSG practice guidelines stress the importance of individualising treatment decisions around pregnancy after full discussion with the patient. ${ }^{19}$ Mycophenelate should be avoided in pregnancy, as it is potentially teratogenic.

\section{CONCLUSIONS AND UNANSWERED QUESTIONS IN AIH}

Happily, standard therapy controls the majority of disease in patients with AIH. However some key clinic questions remain. First, the exact role of budesonide in induction and fine-tuning strategies in maintenance therapy require further elucidation. However, budesonide is limited in cirrhotic patients and, therefore, standard therapy with prednis(ol)one is preferred. Second, defining what constitutes remission in $\mathrm{AIH}$ is crucial in enabling an evidence-based approach to consideration of withdrawal of pharmacological therapy. Last, the issue of treatment failure remains problematic, as a standardised evidence-based approach which delineates the role of second-line agents does not currently exist. However, current BSG guidelines include a suggested treatment algorithm for the management of treatment failure. ${ }^{19}$ For those patients who do progress to cirrhosis, a subset may develop indications for liver transplantation and may expect excellent survival rates following transplantation. Early referral to a transplant centre is recommended for those patients developing treatment failure, portal hypertension or synthetic dysfunction.

Acknowledgements The authors would like to thank Dr Yoh Zen for providing photomicrographs of liver histology.

Contributors SK wrote the article. MH provided conceptual advice in addition to editing and review of the article.

Competing interests None.

Provenance and peer review Not commissioned; externally peer reviewed.

Correction notice This article has been corrected since it was published Online First. On page 6, in the section entitled Long-term management, the sentence "A large retrospective study of AIH patients in Canada demonstrated 10 -year rates of liver-related deaths, 
or requirement for liver transplantation of $94 \%$ in non-cirrhotic versus $67 \%$ for cirrhotic patients, respectively" should read: "A large retrospective study of AIH patients in Canada demonstrated 10-year survival rates to liver-related endpoints of death or liver transplantation were $94 \%$ in non-cirrhotic versus $67 \%$ in cirrhotic patients."

\section{REFERENCES}

1 Invernizzi P. Geoepidemiology of autoimmune liver diseases. J Autoimmun 2010;34:J300-6.

2 Cook GC, Mulligan R, Sherlock S. Controlled prospective trial of corticosteroid therapy in active chronic hepatitis. Q J Med 1971;40:159-85.

3 Bogdanos DP, Lenzi M, Okamoto M, et al. Multiple viral/self immunological cross-reactivity in liver kidney microsomal antibody positive hepatitis $\mathrm{C}$ virus infected patients is associated with the possession of HLA B51. Int J Immunopathol Pharmacol 2004;17:83-92.

4 Kerkar N, Choudhuri K, Ma Y, et al. Cytochrome P4502D6 (193-212): a new immunodominant epitope and target of virus/self cross-reactivity in liver kidney microsomal autoantibody type 1-positive liver disease. J Immunol 2003;170:1481-9.

5 Czaja AJ. Drug-induced autoimmune-like hepatitis. Dig Dis Sci 2011;56:958-76.

6 Longhi MS, Hussain MJ, Mitry RR, et al. Functional study of CD4+CD25 + regulatory T cells in health and autoimmune hepatitis. J Immunol 2006;176:4484-91.

7 Longhi MS, Hussain MJ, Bogdanos DP, et al. Cytochrome P450IID6-specific CD8 T cell immune responses mirror disease activity in autoimmune hepatitis type 2. Hepatology 2007;46:472-84.

8 Ferri S, Longhi MS, De Molo C, et al. A multifaceted imbalance of T cells with regulatory function characterizes type 1 autoimmune hepatitis. Hepatology 2010;52:999-1007.

9 Longhi MS, Liberal R, Holder B, et al. Inhibition of Interleukin-17 promotes differentiation of CD25(-) cells into stable T regulatory cells in patients with autoimmune hepatitis. Gastroenterology 2012;142:1526-35.

10 Al-Chalabi T, Boccato S, Portmann BC, et al. Autoimmune hepatitis (AIH) in the elderly: a systematic retrospective analysis of a large group of consecutive patients with definite AIH followed at a tertiary referral centre. $J$ Hepatol 2006;45:575-83.

11 Ngu JH, Bechly K, Chapman BA, et al. Population-based epidemiology study of autoimmune hepatitis: a disease of older women? J Gastroenterol Hepatol 2010;25:1681-6.

12 Werner M, Prytz H, Ohlsson B, et al. Epidemiology and the initial presentation of autoimmune hepatitis in Sweden: a nationwide study. Scand J Gastroenterol 2008;43:1232-40.

13 Ferrari R, Pappas G, Agostinelli D, et al. Type 1 autoimmune hepatitis: patterns of clinical presentation and differential diagnosis of the 'acute' type. QJM 2004;97:407-12.

14 Lee WM, Squires RH Jr, Nyberg SL, et al. Acute liver failure: summary of a workshop. Hepatology 2008;47:1401-15.

15 Brandsaeter B, Hockerstedt K, Friman S, et al. Fulminant hepatic failure: outcome after listing for highly urgent liver transplantation-12 years experience in the nordic countries. Liver Transpl 2002;8:1055-62.

16 Escorsell A, Mas A, de la Mata M. Acute liver failure in Spain: analysis of 267 cases. Liver Transpl 2007;13:1389-95.
17 Gregorio GV, Portmann B, Reid F, et al. Autoimmune hepatitis in childhood: a 20-year experience. Hepatology 1997;25:541-7.

18 Verma S, Torbenson M, Thuluvath PJ. The impact of ethnicity on the natural history of autoimmune hepatitis. Hepatology 2007;46:1828-35.

19 Gleeson D, Heneghan MA. British Society of Gastroenterology (BSG) guidelines for management of autoimmune hepatitis. Gut 2011;60:1611-29.

20 Czaja AJ. Performance parameters of the conventional serological markers for autoimmune hepatitis. Dig Dis Sci 2011;56:545-54.

21 Czaja AJ. Autoantibodies as prognostic markers in autoimmune liver disease. Dig Dis Sci 2010;55:2144-61.

22 Alvarez F, Berg PA, Bianchi FB, et al. International Autoimmune Hepatitis Group report: review of criteria for diagnosis of autoimmune hepatitis. J Hepatol 1999;31: 929-38.

23 Suzuki A, Brunt EM, Kleiner DE, et al. The use of liver biopsy evaluation in discrimination of idiopathic autoimmune hepatitis versus drug-induced liver injury. Hepatology 2011;54:931-9.

24 Yasui S, Fujiwara K, Yonemitsu Y, et al. Clinicopathological features of severe and fulminant forms of autoimmune hepatitis. J Gastroenterol 2011;46:378-90.

25 Johnson PJ, McFarlane IG. Meeting report: International Autoimmune Hepatitis Group. Hepatology 1993;18:998-1005.

26 Hennes EM, Zeniya M, Czaja AJ, et al. Simplified criteria for the diagnosis of autoimmune hepatitis. Hepatology 2008;48:169-76.

27 Czaja AJ. Performance parameters of the diagnostic scoring systems for autoimmune hepatitis. Hepatology 2008;48:1540-8.

28 Muratori P, Granito A, Pappas G, et al. Validation of simplified diagnostic criteria for autoimmune hepatitis in Italian patients. Hepatology 2009;49:1782-3; author reply 83.

29 Miyake Y, Iwasaki Y, Kobashi H, et al. Clinical features of autoimmune hepatitis diagnosed based on simplified criteria of the International Autoimmune Hepatitis Group. Dig Liver Dis 2010;42:210-15.

30 Gatselis NK, Zachou K, Papamichalis P, et al. Comparison of simplified score with the revised original score for the diagnosis of autoimmune hepatitis: a new or a complementary diagnostic score? Dig Liver Dis 2010;42:807-12.

31 Yeoman AD, Westbrook RH, Al-Chalabi T, et al. Diagnostic value and utility of the simplified International Autoimmune Hepatitis Group (IAIHG) criteria in acute and chronic liver disease. Hepatology 2009;50:538-45.

32 Qiu D, Wang Q, Wang H, et al. Validation of the simplified criteria for diagnosis of autoimmune hepatitis in Chinese patients. J Hepatol 2011;54:340-7.

33 Munoz-Espinosa L, Alarcon G, Mercado-Moreira A, et al. Performance of the international classifications criteria for autoimmune hepatitis diagnosis in Mexican patients. Autoimmunity 2011;44:543-8.

34 Fujiwara K, Yasui S, Tawada A, et al. Diagnostic value and utility of the simplified International Autoimmune Hepatitis Group criteria in acute-onset autoimmune hepatitis. Liver Int 2011;31:1013-20.

35 Lohse AW, Wiegard C. Diagnostic criteria for autoimmune hepatitis. Best Pract Res Clin Gastroenterol 2011;25: $665-71$. 
36 Boberg KM, Chapman RW, Hirschfield GM, et al. Overlap syndromes: the International Autoimmune Hepatitis Group (IAIHG) position statement on a controversial issue. J Hepatol 2011;54:374-85.

37 European Association for the Study of the Liver. Clinical Practice Guidelines: management of cholestatic liver diseases. J Hepatol 2009;51:237-67.

38 Gregorio GV, Portmann B, Karani J, et al. Autoimmune hepatitis/sclerosing cholangitis overlap syndrome in childhood: a 16-year prospective study. Hepatology 2001;33:544-53.

39 Kamisawa T, Funata N, Hayashi Y, et al. A new clinicopathological entity of IgG4-related autoimmune disease. J Gastroenterol 2003;38:982-4.

40 Chung $\mathrm{H}$, Watanabe T, Kudo M, et al. Identification and characterization of IgG4-associated autoimmune hepatitis. Liver Int 2010;30:222-31.

41 Umemura T, Zen Y, Hamano $\mathrm{H}$, et al. Clinical significance of immunoglobulin G4-associated autoimmune hepatitis. J Gastroenterol 2011;46(Suppl 1):48-55.

42 Manns MP, Czaja AJ, Gorham JD, et al. Diagnosis and management of autoimmune hepatitis. Hepatology 2010;51:2193-213.

43 Lamers M, van Oijen M, Pronk M, et al. Treatment options for autoimmune hepatitis: a systematic review of randomized controlled trials. J Hepatol 2010;53:191-8.

44 Johnson PJ, McFarlane IG, Williams R. Azathioprine for long-term maintenance of remission in autoimmune hepatitis. N Engl J Med 1995;333:958-63.

45 DePinho RA, Goldberg CS, Lefkowitch JH. Azathioprine and the liver. Evidence favoring idiosyncratic, mixed cholestatic-hepatocellular injury in humans. Gastroenterology 1984;86:162-5.

46 Manns MP, Woynarowski M, Kreisel W, et al. Budesonide induces remission more effectively than prednisone in a controlled trial of patients with autoimmune hepatitis. Gastroenterology 2010;139:1198-206.

47 Lohse AW, Gil H. Reactivation of autoimmune hepatitis during budesonide monotherapy, and response to standard treatment. J Hepatol 2011;54:837-9.

48 Montano-Loza AJ, Carpenter HA, Czaja AJ. Improving the end point of corticosteroid therapy in type 1 autoimmune hepatitis to reduce the frequency of relapse. Am J Gastroenterol 2007;102:1005-12.

49 Miyake Y, Iwasaki Y, Terada R, et al. Persistent normalization of serum alanine aminotransferase levels improves the prognosis of type 1 autoimmune hepatitis. J Hepatol 2005;43:951-7.

50 Al-Chalabi T, Heneghan MA. Remission in autoimmune hepatitis: what is it, and can it ever be achieved? Am J Gastroenterol 2007;102:1013-15.

51 Czaja AJ, Wolf AM, Baggenstoss AH. Laboratory assessment of severe chronic active liver disease during and after corticosteroid therapy: correlation of serum transaminase and gamma globulin levels with histologic features. Gastroenterology 1981;80:687-92.

52 Kanzler S, Gerken G, Löhr H, et al. Duration of immunosuppressive therapy in autoimmune hepatitis. J Hepatol 2001;34:354-5.

53 Verma S, Gunuwan B, Mendler M, et al. Factors predicting relapse and poor outcome in type I autoimmune hepatitis: role of cirrhosis development, patterns of transaminases during remission and plasma cell activity in the liver biopsy. Am J Gastroenterol 2004;99:1510-16.
54 Baeres M, Herkel J, Czaja AJ, et al. Establishment of standardised SLA/LP immunoassays: specificity for autoimmune hepatitis, worldwide occurrence, and clinical characteristics. Gut 2002;51:259-64.

55 Heneghan M. Current and novel immunosuppressive therapy for autoimmune hepatitis. Hepatology 2002;35:7-13.

56 Garcia-Buey L, Moreno-Otero R. Mycophenolate mofetil for patients with autoimmune hepatitis and overlap syndromes. Aliment Pharmacol Ther 2011;34:682-4; author reply $84-5$.

57 Zachou K, Gatselis N, Papadamou G, et al. Mycophenolate for the treatment of autoimmune hepatitis: prospective assessment of its efficacy and safety for induction and maintenance of remission in a large cohort of treatment-naive patients. J Hepatol 2011;55:636-46.

58 Alvarez F, Ciocca M, Canero-Velasco C, et al. Short-term cyclosporine induces a remission of autoimmune hepatitis in children. J Hepatol 1999;30:222-7.

59 Van Thiel DH, Wright H, Carroll P, et al. Tacrolimus: a potential new treatment for autoimmune chronic active hepatitis: results of an open-label preliminary trial. Am J Gastroenterol 1995;90:771-6.

60 Heneghan MA, Rizzi P, McFarlane I, et al. Low dose tacrolimus as treatment of severe autoimmune hepatitis: potential role in remission induction. Gut 1999; 44(Suppl 1):A61.

61 Aqel BA, Machicao V, Rosser B, et al. Efficacy of tacrolimus in the treatment of steroid refractory autoimmune hepatitis. J Clin Gastroenterol 2004;38:805-9.

62 Chatur N, Ramji A, Bain VG, et al. Transplant immunosuppressive agents in non-transplant chronic autoimmune hepatitis: the Canadian association for the study of liver (CASL) experience with mycophenolate mofetil and tacrolimus. Liver Int 2005;25:723-7.

63 Larsen FS, Vainer B, Eefsen M, et al. Low-dose tacrolimus ameliorates liver inflammation and fibrosis in steroid refractory autoimmune hepatitis. World J Gastroenterol 2007;13:3232-6.

64 Kainz A, Harabacz I, Cowlrick IS, et al. Review of the course and outcome of 100 pregnancies in 84 women treated with tacrolimus. Transplantation 2000;70:1718-21.

65 Jain AB, Reyes J, Marcos A, et al. Pregnancy after liver transplantation with tacrolimus immunosuppression: a single center's experience update at 13 years. Transplantation 2003;76:827-32.

66 Christopher V, Al-Chalabi T, Richardson PD, et al. Pregnancy outcome after liver transplantation: a single-center experience of 71 pregnancies in 45 recipients. Liver Transpl 2006;12:1138-43.

67 Burak KW, Urbanski SJ, Swain MG. Successful treatment of refractory type 1 autoimmune hepatitis with methotrexate. J Hepatol 1998;29:990-3.

68 Sultan MI, Biank VF, Telega GW. Successful treatment of autoimmune hepatitis with methotrexate. J Pediatr Gastroenterol Nutr 2011;52:492-4.

69 Barth E, Clawson J. A case of autoimmune hepatitis treated with rituximab. Case Reports in Gastroenterology 2010;4:502-9.

70 Grasland A, Sterpu R, Boussoukaya S, et al. Autoimmune hepatitis induced by adalimumab with successful switch to abatacept. Eur J Clin Pharmacol 2012;68:895-8.

71 Feld JJ, Dinh H, Arenovich T, et al. Autoimmune hepatitis: effect of symptoms and cirrhosis on natural history and outcome. Hepatology 2005;42:53-62. 
72 Hoeroldt B, McFarlane E, Dube A, et al. Long-term outcomes of patients with autoimmune hepatitis managed at a nontransplant center. Gastroenterology 2011;140:1980-9.

73 Tan P, Marotta P, Ghent C, et al. Early treatment response predicts the need for liver transplantation in autoimmune hepatitis. Liver Int 2005;25:728-33.

74 Yeoman AD, Al-Chalabi T, Karani JB, et al. Evaluation of risk factors in the development of hepatocellular carcinoma in autoimmune hepatitis: Implications for follow-up and screening. Hepatology 2008;48:863-70.

75 Wong RJ, Gish R, Frederick T, et al. Development of hepatocellular carcinoma in autoimmune hepatitis patients: a case series. Dig Dis Sci 2011;56:578-85.

76 Montano-Loza AJ, Carpenter HA, Czaja AJ. Predictive factors for hepatocellular carcinoma in type 1 autoimmune hepatitis. Am J Gastroenterol 2008;103:1944-51.

77 Migita K, Watanabe Y, Jiuchi Y, et al. Hepatocellular carcinoma and survival in patients with autoimmune hepatitis (Japanese National Hospital Organization-autoimmune hepatitis prospective study). Liver Int 2012;32:837-44.

78 Schramm C, Bubenheim M, Adam R, et al. Primary liver transplantation for autoimmune hepatitis: a comparative analysis of the European Liver Transplant Registry. Liver Transpl 2010;16:461-9.

79 Roberts MS, Angus DC, Bryce CL, et al. Survival after liver transplantation in the United States: a disease-specific analysis of the UNOS database. Liver Transpl 2004;10:886-97.

80 Yeoman AD, Westbrook RH, Zen Y, et al. Early predictors of corticosteroid treatment failure in icteric presentations of autoimmune hepatitis. Hepatology 2011;53:926-34.

81 Ichai P, Duclos-Vallee JC, Guettier C, et al. Usefulness of corticosteroids for the treatment of severe and fulminant forms of autoimmune hepatitis. Liver Transpl 2007;13:996-1003.

82 Neuberger J, Gimson A, Davies M, et al. Selection of patients for liver transplantation and allocation of donated livers in the UK. Gut 2008;57:252-7.

83 Long MD, Herfarth $\mathrm{HH}$, Pipkin CA, et al. Increased risk for non-melanoma skin cancer in patients with inflammatory bowel disease. Clin Gastroenterol Hepatol 2010;8:268-74.

84 Kandiel A, Fraser AG, Korelitz BI, et al. Increased risk of lymphoma among inflammatory bowel disease patients treated with azathioprine and 6-mercaptopurine. Gut $2005 ; 54: 1121-5$

85 Masunaga Y, Ohno K, Ogawa R, et al. Meta-analysis of risk of malignancy with immunosuppressive drugs in inflammatory bowel disease. Ann Pharmacother 2007;41:21-8.

86 Vos AC, Bakkal N, Minnee RC, et al. Risk of malignant lymphoma in patients with inflammatory bowel diseases: a Dutch nationwide study. Inflamm Bowel Dis 2011;17:1837-45.

87 Meggitt SJ, Anstey AV, Mohd Mustapa MF, et al. British Association of Dermatologists' guidelines for the safe and effective prescribing of azathioprine 2011. Br J Dermatol 2011;165:711-34

88 Harpreet Kaur D, Rory A, Louise TE, et al. Clinical significance of azathioprine metabolites for the maintenance of remission in autoimmune hepatitis. Hepatology 2012 Apr 5. doi: 10.1002/hep.25760. [Epub ahead of print].

89 Gisbert JP, Gomollon F, Cara C, et al. Thiopurine methyltransferase activity in Spain: a study of 14,545 patients. Dig Dis Sci 2007;52:1262-9.

90 Heneghan MA, Allan ML, Bornstein JD, et al. Utility of thiopurine methyltransferase genotyping and phenotyping, and measurement of azathioprine metabolites in the management of patients with autoimmune hepatitis. J Hepatol 2006;45:584-91.

91 Czaja AJ, Carpenter HA. Thiopurine methyltransferase deficiency and azathioprine intolerance in autoimmune hepatitis. Dig Dis Sci 2006;51:968-75.

92 Hindorf U, Jahed K, Bergquist A, et al. Characterisation and utility of thiopurine methyltransferase and thiopurine metabolite measurements in autoimmune hepatitis. J Hepatol 2010;52:106-11.

93 Steven MM, Buckley JD, Mackay IR. Pregnancy in chronic active hepatitis. Q J Med 1979;48:519-31.

94 Heneghan MA, Norris SM, O’Grady JG, et al. Management and outcome of pregnancy in autoimmune hepatitis. Gut 2001;48:97-102.

95 Buchel E, Van Steenbergen W, Nevens F, et al. Improvement of autoimmune hepatitis during pregnancy followed by flare-up after delivery. Am J Gastroenterol 2002;97: 3160-5.

96 Schramm C, Herkel J, Beuers U, et al. Pregnancy in autoimmune hepatitis: outcome and risk factors. $A m \mathrm{~J}$ Gastroenterol 2006;101:556-60.

97 Terrabuio DR, Abrantes-Lemos CP, Carrilho FJ, et al. Follow-up of pregnant women with autoimmune hepatitis: the disease behavior along with maternal and fetal outcomes. J Clin Gastroenterol 2009;43:350-6.

98 Westbrook RH, Yeoman AD, Kriese S, et al. Outcomes of pregnancy in women with autoimmune hepatitis. J Autoimmun 2012;38:J239-44.

99 Francella A, Dyan A, Bodian C, et al. The safety of 6-mercaptopurine for childbearing patients with inflammatory bowel disease: a retrospective cohort study. Gastroenterology 2003;124:9-17. 